\title{
Mycorrhiza improves plant growth and photosynthetic characteristics of tea plants in response to drought stress
}

\author{
Fengjun DAI ${ }^{1}$; Ziyi RONG ${ }^{1}$; Qiangsheng WU ${ }^{1}$; Elsayed Fathi ABD_AllaH ${ }^{3}$; Chunyan LIU ${ }^{1,2, *}$; Shengrui LIU ${ }^{2, *}$ \\ ${ }^{1}$ College of Horticulture and Gardening, Yangtze University, Jingzhou, 434025, China \\ ${ }^{2}$ State Key Laboratory of Tea Plant Biology and Utilization, Anhui Agricultural University, Hefei, 230036, China \\ 3 Plant Production Department, Faculty of Food and Agricultural Sciences, King Saud University, Riyadh, 11451, Saudi Arabia
}

Key words: Arbuscular mycorrhizal fungi, Drought stress, Photosynthesis, Tea

\begin{abstract}
Tea plants are sensitive to soil moisture deficit, with the level of soil water being a critical factor affecting their growth and quality. Arbuscular mycorrhizal fungi (AMF) can improve water and nutrient absorption, but it is not clear whether AMF can improve the photosynthetic characteristics of tea plants. A potted study was conducted to determine the effects of Claroideoglomus etunicatum on plant growth, leaf water status, pigment content, gas exchange, and chlorophyll fluorescence parameters in Camellia sinensis cv. Fuding Dabaicha under well-watered (WW) and drought stress (DS) conditions. Root mycorrhizal colonization and soil hyphal length were significantly reduced by the eightweek DS treatment. AMF inoculation displayed a significant increase in shoot and root biomass production. The relative water content, leaf water potential, nitrogen balance index, pigment content, maximum photometric effect (Fv/Fm, QY_max), and steady-state photometric effect Y (II) (QY_Lss) decreased dramatically, while the leaf water saturation deficit and steady-state non-photochemical fluorescence quenching (NPQ_Lss) generally increased under DS conditions. Mycorrhizal treatment induced significantly higher relative water content, leaf water potential, nitrogen balance index, pigment (chlorophyll, flavonoid, and anthocyanin) content, net photosynthesis rate, transpiration rate, stomatal conductance, intercellular $\mathrm{CO}_{2}$ concentration, QY_max, and QY_Lss; however, it resulted in a lower leaf water saturation deficit and NPQ_Lss under both WW and DS conditions, as compared with nonmycorrhizal plants. These results imply that AMF promoted tea plant growth and alleviated negative effects of DS by promoting gas exchange, regulating the water status of leaves, and regulating photosynthetic parameters.
\end{abstract}

\section{Introduction}

Arbuscular mycorrhizal fungi (AMF) are widespread in soils and can establish symbioses with most vascular plants, thus influencing the soil microhabitat around plant roots and the forms of soil mineral nutrients present, and driving soil nutrient cycling (Cheng et al., 2021; Meng et al., 2020). AMF can improve plant growth and development, root uptake capacity, stress tolerance, osmotic adjustment, and antioxidant capacity (Zou et al., 2021). In addition, symbiosis between AMF and plant roots can improve crop yield and significantly contribute to plant growth and development, morphological establishment, and yield increases under adversity stress (Saboor et al., 2021).

*Address correspondence to: Chunyan Liu, 201573031@yangtzeu.edu.cn; Shengrui Liu, liushengrui@ahau.edu.cn

Received: 23 August 2021; Accepted: 26 September 2021
Tea plant (Camellia sinensis (L.) O. Ktze.) is a perennial evergreen woody plant in the Camelliaceae family. It is one of the important industrial crops in South Asia, and its growth and development are strictly tied to the external growth environment (Qian et al., 2018). However, increasing temperature and frequency of drought in recent years pose a great threat to the growth of tea plants (Gupta et al., 2013). Drought stress (DS) increases water loss rate (WLR) and decreases relative water content (RWC), dry mass, chlorophyll, carotenoid, and total leaf phenolic content and antioxidants such as ascorbate and glutathione in tea (Upadhyaya and Panda, 2013). In 2009, the decrease in tea yield caused by DS is as high as $31 \%$ of tea plantations in Sri Lanka, and the plant mortality caused by drought and clonal variation is $6 \%-19 \%$ (Cheruiyot et al., 2009). Chen et al. (2019) reported that Australian white tea grew slowly and accompanied with less resistant under natural drought conditions. In addition, Wang et al. (2020) found that tea plants had smaller leaf area, fewer leaves, lower fresh and 
dry weight of tea plants, weaker photosynthesis, and lower yields under different moisture conditions (soil moisture content of $15 \%-25 \%$ and a total of 20 days of drought). In order to cope with DS, tea plants have a series of coping mechanisms in the process of growth, photosynthesis, and metabolism, primarily related to changes in leaf structure, chlorophyll content, chlorophyll fluorescence parameters, and soluble sugars (Upadhyaya and Panda, 2013).

Establishing symbiosis with AMF to improve nutrient status and water demand, photosynthesis, and other parameters required to cope with DS could be a superior strategy. Under natural cultivation conditions, a variety of AMF are widely present in tea plantations, especially in red soil (Wu et al., 2019). A previous study showed that AMF had an important impact on nutrient uptake and physiological ecology in tea plants, manifested by increased chlorophyll content and leaf area, and reduced leaf water potential, thus enhancing plant water absorption capacity (Mathur et al., 2019). It can also significantly increase the mass fraction of proline, soluble protein, and glutathione glycopeptides, as well as the contents of various mineral nutrients required by tea plants, thereby improving the quality of tea (Cao et al., 2021). Based on these reports, it is of great importance to promote the positive effects of AMF on tea tree growth and to further research the effects of AMF on tea growth and quality under drought conditions, as well as the mechanisms of AMF on tea resilience.

Hence, the purposes of this study were to (i) to analyze the effects of AMF inoculation on the growth, development, and photosynthetic characteristics of tea plants under DS, and (ii) to clarify the potential mechanisms of AMF-induced drought resistance in host plants.

\section{Materials and Methods}

\section{Plant culture}

The AM fungus used in this experiment was Clariodeoglous etunicatum (Nicol. \& Gerd.) Schüßler \& Walker [BGC GZ03C]. The selection of strains was based on the results of Shao et al. (2018). C. etunicatum was provided by the Bank of Glomeromycota in China (BGC) and expanding propagation in white clover (Trifolium repens) for 16 weeks.

The test plants were Camellia sinensis cv. 'Fuding Dabaicha'. The seeds were supplied by the Tea Research Institute of the Guizhou Academy of Agricultural Sciences. The seeds were disinfected and germinated for 30 days, according to Liu et al. (2020). Then, two 3-leaf-old tea seedlings in uniform size were transplanted into a $1.8 \mathrm{~L}$ plastic pot, each pot was prefilled with $1.5 \mathrm{~kg}$ sterilized $\left(0.11 \mathrm{MPa}, 121^{\circ} \mathrm{C}, 2 \mathrm{~h}\right)$ mixture substance (soil: sand = $1: 1, \mathrm{v} / \mathrm{v})$. At seedling transporting, an AMF inoculum of 80 g C. etunicatum carrying 1500 spores, and some colonized root segments of white clover were inoculated with the growth substrates for AMF treatments. The non-AMFinoculated treatment was supplied with the same amount of sterilized inoculum plus $2 \mathrm{~mL}$ filtrate $(25-\mu \mathrm{m}$ filter $)$ of mycorrhizal inoculum to minimize differences in other microbial communities. All the tea seedlings were evenly placed in plastic greenhouse conditions $\left(900 \mu \mathrm{mol} / \mathrm{m}^{2} / \mathrm{s}\right.$ photon flux density, $28 / 20^{\circ} \mathrm{C}$ day/night temperature, and $80 \%$ relative humidity) in Yangtze University (Jingzhou, China).
After transportation, all the AMF and non-AMF seedlings grew for 6 weeks under the conditions of the soil being at $75 \%$ maximum water holding capacity (soil WW status). Subsequently, half of the seedlings were maintained under WW conditions for 8 weeks, and the remaining half seedlings, including AMF and non-AMF treatments, were exposed to $55 \%$ of the maximum water holding capacity of the soil (DS status) for 8 weeks. Before tea seedling transplanting, we pre-calculated the actual soil water content and the maximum field water capacity by weighing method, thus calculated the soil weight of each pot under WW and DS conditions. And then the pots were maintained at a stable water content by weighing the water content daily at 18:00 pm, and the amount of water lost was restored to the designated soil water levels.

\section{Experimental design}

The experiment was conducted in a $2 \times 2$ two-factorial experimental design with four treatments. Factor 1 was inoculated with (+AMF) or without C. etunicatum (-AMF). Factor 2 was moisture treatment, having either $75 \%$ of maximum soil water holding capacity (soil WW status) or $55 \%$ of maximum soil water holding capacity (soil DS status). The four treatments were WW + AMF, WW, DS + AMF, and DS. Each treatment was replicated six times in a total of 24 pots, completely randomly arranged.

\section{Variable determinations}

Before these seedlings were harvested, the third top leaf in full expedition was selected to measure the photosynthetic parameters using a Li-6400 portable photosynthesis system (LICOR Biosciences, Lincoln, NE, USA) to obtain the net photosynthetic rate $(P n)$, intercellular $\mathrm{CO}_{2}$ concentration $(C i)$, stomatal conductance $(G s)$, and transpiration rate $(\mathrm{Tr})$ under the condition of $400 \mu \mathrm{mol} / \mathrm{mol}\left[\mathrm{CO}_{2}\right]$ on a sunny morning from approximately 09:00 to 11:00 am. Subsequently, chlorophyll, anthocyanin, flavonoid content, and nitrogen balance index were measured using a Dualex portable plant polyphenol-chlorophyll meter (Force-A, Orsay, France). Further, fully expanded functional leaves were randomly selected to determine the chlorophyll fluorescence parameters, including maximum photometric effect (Fv/Fm, QY_max), steady-state photometric effect Y (II) (QY_Lss), and steady-state nonphotochemical fluorescence quenching (NPQ_Lss) by the domestic RAP-FLUO high-throughput plant fluorescence phenotype monitoring platform from Gufeng Optoelectronics.

At seedlings harvest, plant height, leaf number, stem diameter (the root neck is about $1 \mathrm{~cm}$ away from the surface of potted soil), and biomass in shoots and roots were recorded. Meanwhile, a fully expanded leaf was randomly selected to measure the relative water content (RWC) according to Liu et al. (2020), and the water saturation deficit was calculated (1-relative water content). The leaf water potential was measured using a PSYPRO water potential system with a leaf hygrometer (L-51A-SF, WESCOR Inc.).

The root systems in different levels were then randomly selected, the root tips were removed, and approximately 40 root segments (1-cm long) from each treatment were stained with $0.05 \%(\mathrm{w} / \mathrm{v})$ trypan blue in lactophenol for mycorrhizal status evaluation (Phillips and Hayman, 1970). 
Mycorrhizal colonization rate $(\%)=$ length of the colonized root segment/length of the observed root segment $\times 100$. In addition, plant harvest and fresh rhizosphere soil samples were collected to determine the soil hyphae length using the method described by Bethlenfalvay and Ames (1987).

\section{Statistical analysis}

The ANOVA procedure of SAS (8.1) software was used to test for differences between treatments, and multiple comparison analysis was performed using Duncan's new complex polar difference method $(P<0.05)$.

\section{Results}

Changes in mycorrhiza development and plant growth Roots of tea seedlings were colonized by C. etunicatum, establishing a good symbiotic relationship and hyphal network in the rhizosphere. In contrast, DS inhibited mycorrhizal infection by $74.83 \%$ and the establishment of mycelial network by $42.42 \%$ (Table 1 ). In addition, DS significantly decreased plant height in AMF-colonized seedlings and stem diameter in both AMF and non-AMF seedlings. AM-colonized seedlings showed $17.28 \%$ and $10.30 \%$ higher in plant height and stem diameter under WW, and $22.22 \%$ higher in leaf numbers under DS condition than that in non-AMF colonized seedlings, respectively (Table 1).

\section{Changes in biomass production}

Compared with WW, DS significantly reduced the tea stem fresh weight by $61.54 \%$ in mycorrhizal treatments, but it had no significant effect on leaf and root fresh weight (Fig. 1). However, inoculation with AMF dramatically increased the fresh weight in leaves, stems and roots were by $26.55 \%, 65.79 \%$ and $36.96 \%$ under WW, and $30.48 \%$, $21.87 \%$ and $32.24 \%$ under DS, respectively, as compared with non-AMF inoculation seedlings.

\section{Changes in leaf moisture status}

The results showed a decrease in leaf water potential and an increase in water saturation deficit caused by DS, irrespective of AMF inoculated or not. Meanwhile, the leaf RWC was $4.80 \%$ lower in non-AMF seedlings under DS than in WW (Fig. 2). Whereas, mycorrhizal inoculation with C. etunicatum substantially increased the leaf water potential and RWC by $25.81 \%$ and $17.07 \%$ and $11.90 \%$ and $13.75 \%$ under WW and DS conditions, respectively. Moreover, lower

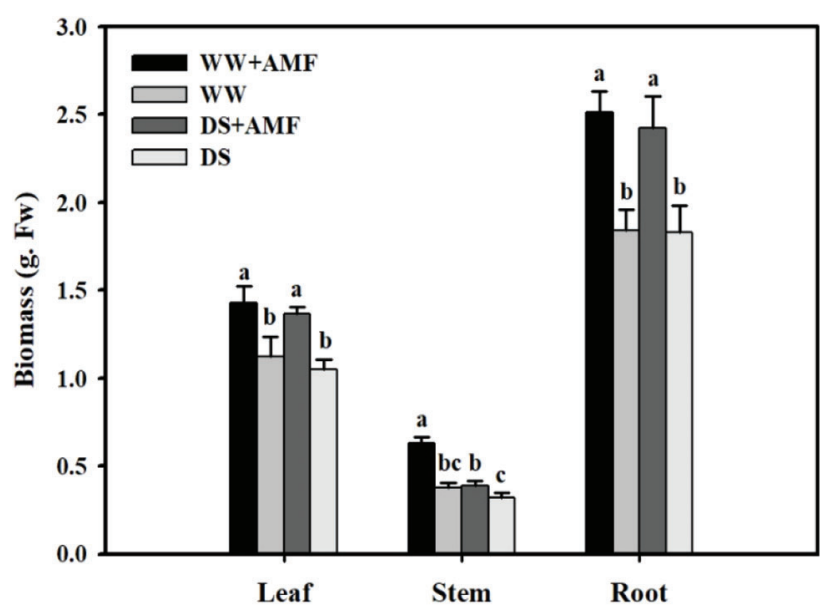

FIGURE 1. Effect of AMF on biomass of tea (Camellia sinensis 'Fuding Dabaicha') seedlings under drought stress. Data (means \pm $\mathrm{SD}, \mathrm{N}=6$ ) with different letter above bars indicated significant differences between treatments at the $5 \%$ level. The same as below.

water saturation deficit was memorably appeared in mycorrhiza seedlings ignorance of water status (Fig. 2).

Changes in pigment content and nitrogen balance index Compared with WW treatment, DS generally decreased the nitrogen balance index (NBI) in both AMF- and non-AMF seedlings (Fig. 3a). C. etunicatum inoculation meaningfully increased the leaf NBI of tea seedlings by $30.61 \%$ and $24.46 \%$ under WW and DS conditions, respectively. Mycorrhizal seedlings exhibited considerably higher contents of chlorophyll and flavonoids than the non-AMF seedlings under WW and DS conditions (Figs. $3 \mathrm{~b}$ and $3 \mathrm{c}$ ). In addition, inoculation with AMF substantially increased the anthocyanin content in WW, but not in the DS treatment (Fig. 3d).

\section{Changes in gas exchange}

DS considerably decreased $G s$ and $C i$ but did not affect $P n$ and $\operatorname{Tr}$ regardless of AMF treated or not. Compared with nonAMF treatment, inoculation with C. etunicatum remarkably increased the $P n, G s, C i$, and $\operatorname{Tr}$ by $79.37 \%, 79.16 \%, 32.12 \%$, and $98.92 \%$ under WW and $82.46 \%, 96.91 \%, 39.32 \%$, and $112.93 \%$ under DS, respectively (Fig. 4).

Changes in chlorophyll fluorescence

Leaf chlorophyll fluorescence parameters of tea leaves were observed (Fig. 5). Compared to WW treatment, DS

TABLE 1

Effects of AMF on the plant growth and mycorrhizal colonization of tea seedlings (Camellia sinensis 'Fuding Dabaicha') under drought stress

\begin{tabular}{llllll}
\hline Treatments & $\begin{array}{l}\text { Mycorrhizal } \\
\text { colonization (\%) }\end{array}$ & $\begin{array}{l}\text { Soil hyphal } \\
\text { length }(\mathbf{c m} / \mathbf{g})\end{array}$ & Plant height $(\mathbf{c m})$ & Stem diameter (mm) & $\begin{array}{l}\text { Leaf number } \\
(\# / \text { plant) }\end{array}$ \\
\hline WW + AMF & $28.76 \pm 2.18 \mathrm{a}$ & $7.12 \pm 0.55 \mathrm{a}$ & $12.35 \pm 0.39 \mathrm{a}$ & $2.57 \pm 0.09 \mathrm{a}$ & $11 \pm 1 \mathrm{a}$ \\
$\mathrm{WW}$ & $0.00 \pm 0.00 \mathrm{c}$ & $0.00 \pm 0.00 \mathrm{c}$ & $10.53 \pm 0.47 \mathrm{~b}$ & $2.33 \pm 0.07 \mathrm{~b}$ & $10 \pm 0 \mathrm{ab}$ \\
DS + AMF & $16.45 \pm 1.42 \mathrm{~b}$ & $4.10 \pm 0.19 \mathrm{~b}$ & $10.43 \pm 0.45 \mathrm{~b}$ & $2.27 \pm 0.03 \mathrm{bc}$ & $11 \pm 0 \mathrm{a}$ \\
DS & $0.00 \pm 0.00 \mathrm{c}$ & $0.00 \pm 0.00 \mathrm{c}$ & $10.06 \pm 0.18 \mathrm{~b}$ & $2.13 \pm 0.08 \mathrm{c}$ & $9 \pm 1 \mathrm{~b}$ \\
\hline
\end{tabular}

Note: Data (mean \pm SD, $N=6$ ) followed by different letters in the same column indicate statistically significant differences at the $5 \%$ probability level. The same as below. 

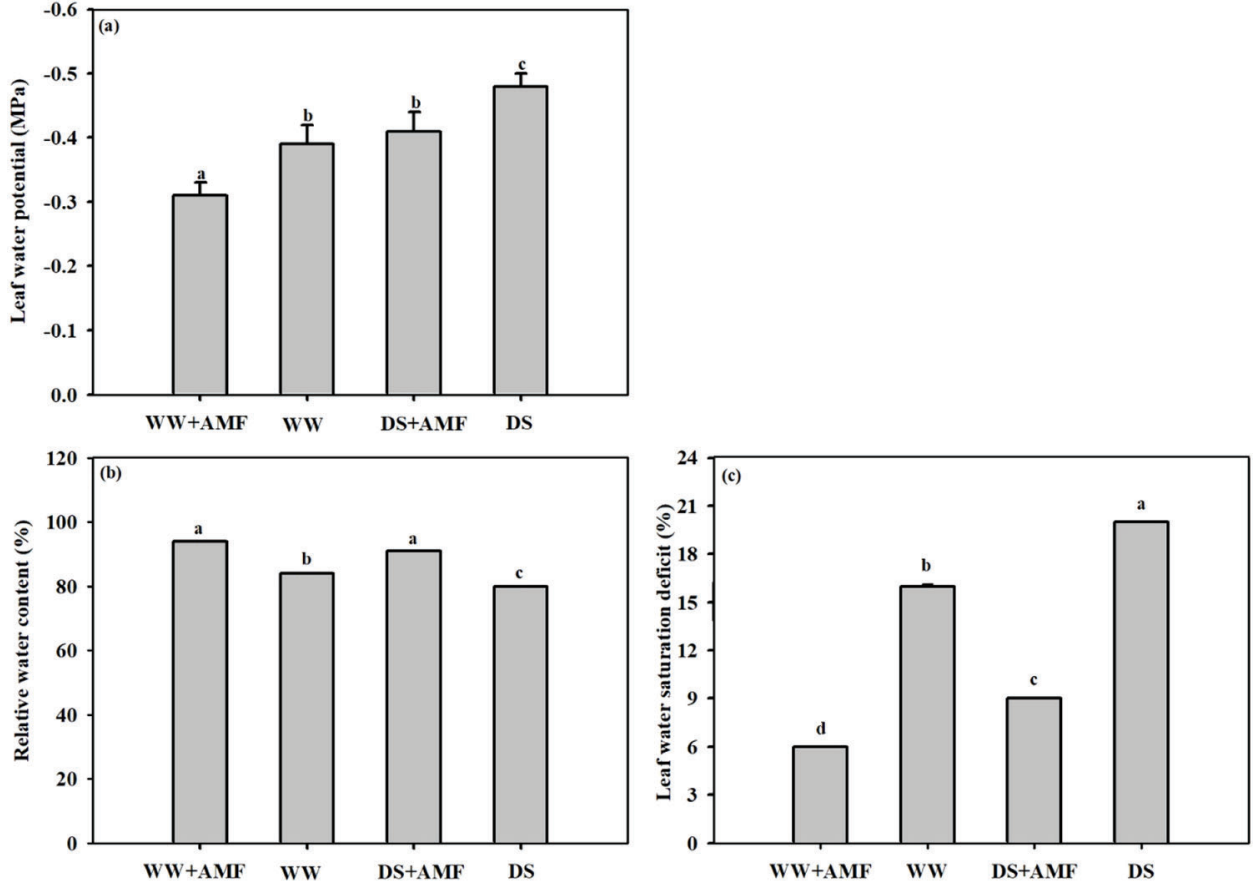

FIGURE 2. Effect of AMF on leaf moisture status of tea (Camellia sinensis 'Fuding Dabaicha') seedlings under drought stress. (a) Leaf water potential, (b) Relatively water content. (c) Leaf water saturation deficit.

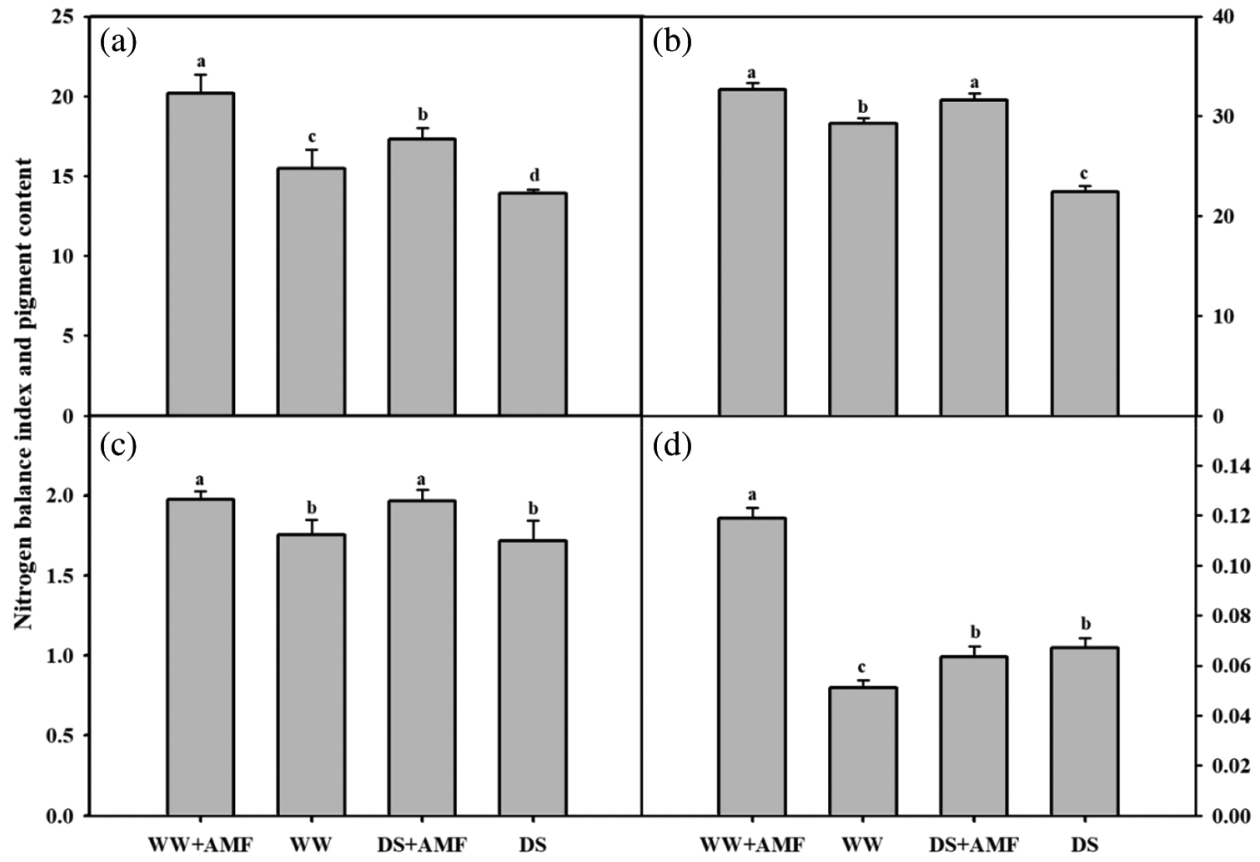

FIGURE 3. Effect of AMF on the leaf nitrogen balance index and photosynthetic pigment content in tea (Camellia sinensis 'Fuding Dabaicha') seedlings under drought stress. (a) Nitrogen balance index (b) Chlorophyll content $(\mathrm{mg} / \mathrm{g})$ (c) Flavonoid content $(\mu \mathrm{g} / \mathrm{g})(\mathrm{d})$ Anthocyanin content $(\mathrm{mg} / \mathrm{g})$.

considerably decreased the leaf QY_max and QY_Lss but increased NPQ_Lss of tea seedlings regardless of AMF treated or not, whereas AMF treatment notably ameliorated these fluorescence parameters (Table 2, Fig. 5). Compared with non-mycorrhizal treatment, AMF seedlings showed significantly higher leaf QY_max, QY_Lss and lower NPQ_Lss by $30.51 \%, 10.34 \%$, and $20.83 \%$ under WW and $41.46 \%, 12.00 \%$, and $35.14 \%$ under DS, separately (Table 2).

\section{Discussion}

Drought is an important factor limiting plant growth, especially tea plants with significantly sensitive to soil water status. Whereas, AMF external hyphae can improve the root activity of host plants and enhance the absorption of soil water and nutrients, thus promoting plant growth and development (Shao et al., 2021). In the present study, C. etunicatum has been shown to colonize tea roots and establish a symbiotic association, which agrees with the findings of Shao et al. (2019) on tea seedlings. Moreover, root mycorrhizal colonization of tea seedlings by C. etunicatum was dramatically inhibited by the 8-week DS treatment. This is consistent with Liu et al. (2020), who observed a decrease in root colonization by C. etunicatum in tea plants exposed to DS. Prolonged DS inhibited spore germination and accumulation of carbohydrates, thus triggering a decrease in AMF colonization in the host plants (Tyagi et al., 2017). 

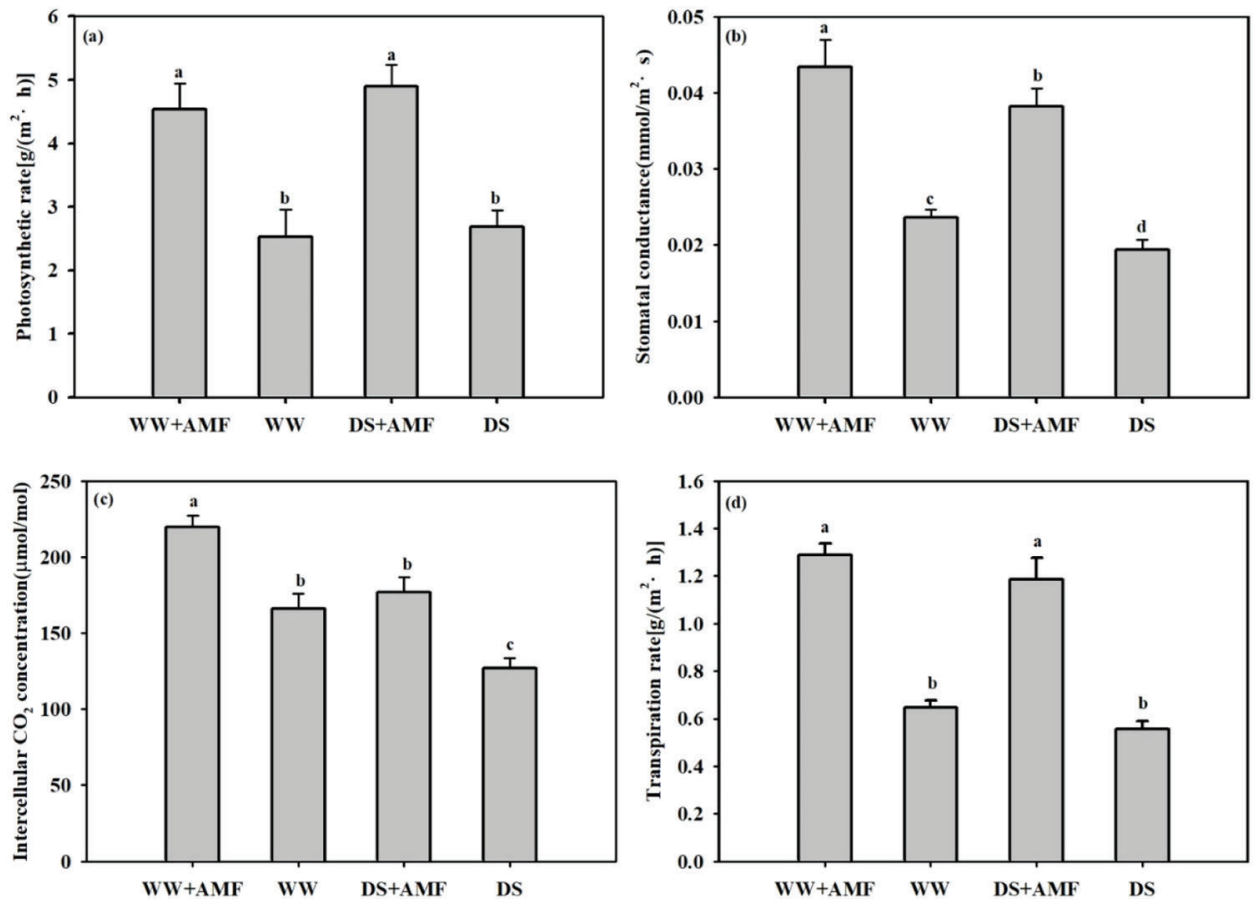

FIGURE 4. Effect of AMF on photosynthetic parameters of tea (Camellia sinensis 'Fuding Dabaicha') seedlings under drought stress. (a) net photosynthetic rate $(\mathrm{Pn})$, (b) stomatal conductance (Gs), (c) intercellular $\mathrm{CO}_{2}$ concentration (Ci), (d), and transpiration rate (Tr).

TABLE 2

Effect of AMF on chlorophyll fluorescence parameters of tea seedlings (Camellia sinensis cv. Fuding Dabaicha) under drought stress

\begin{tabular}{llll}
\hline Treatments & QY_max & QY_Lss & NPQ_Lss \\
\hline WW + AMF & $0.77 \pm 0.04 \mathrm{a}$ & $0.32 \pm 0.02 \mathrm{a}$ & $0.19 \pm 0.01 \mathrm{c}$ \\
WW & $0.59 \pm 0.03 \mathrm{~b}$ & $0.29 \pm 0.01 \mathrm{~b}$ & $0.24 \pm 0.01 \mathrm{~b}$ \\
DS + AMF & $0.58 \pm 0.03 \mathrm{~b}$ & $0.28 \pm 0.01 \mathrm{~b}$ & $0.24 \pm 0.02 \mathrm{~b}$ \\
DS & $0.41 \pm 0.02 \mathrm{c}$ & $0.25 \pm 0.01 \mathrm{c}$ & $0.37 \pm 0.02 \mathrm{a}$ \\
\hline
\end{tabular}

It is well known that DS can reduce soil water supply, decrease root water absorption and plant water content, and inhibit plant growth and development (Liu et al., 2020). AMF can absorb soil water through its widely spread hyphal network and transport it to the plant root, thus improving the water status of host plants (Zhang et al., 2018). A previous study reported that approximately $4 \%$ of water in AMF plant roots originates from mycorrhizal hyphae (He et al., 2019). In this study, DS substantially decreased the leaf water potential and RWC and increased the leaf water saturation deficit, while C. etunicatum inoculation markedly increased the leaf water status of tea seedlings, regardless of the soil water status. These results are in agreement with earlier studies on trifoliate orange and tea seedlings ( $\mathrm{He}$ et al., 2019; Liu et al., 2020). Moreover, mycorrhizal external hyphae can diffuse into the soil beyond the root system to absorb water and nutrients for the plant partner, thus promoting the growth performance of host plants (Zhang et al., 2018). In this study, the growth-promoting effects of mycorrhizal inoculation on tea seedlings were mainly indicated by an increase in biomass, while mycorrhizal inoculation significantly increased biomass in shoots and roots of tea plants, irrespective of soil water status, which was in accordance with the findings of Wu et al. (2016) on trifoliate orange and Quiroga et al. (2020) on Zea mays. Under mycorrhizal treatment, the improvement in plant growth may be due to the enhancement of water and nutrient absorption capacity of extraradical hyphae in AMFcolonized seedlings (He et al., 2019).

Photosynthetic pigments are the key medium for plants to transform inorganic matter into organic matter, which is one of the important indicators reflecting plant photosynthesis and nutrition. The proportion of each component markedly impact the plant photosynthesis, and further affects the normal growth and stress resistance of plants to a certain extent (Mathur et al., 2019). Flavonoids are secondary metabolites found in many plants. They have strong antioxidant capacity, can improve plants resistance against various stresses, eliminate reactive oxygen species, and protect plant cells (Tohge et al., 2017). Previous study reported that DS could affect the photosynthetic capacity of plants and reduce the photosynthetic pigment content in leaves (Lin et al., 2014), while inoculation with AMF can reduce the negative effects caused by DS and increase the photosynthetic pigment content (Xie et al., 2019). In this study, higher contents of chlorophyll, flavonoids, and anthocyanins were observed in AMF seedlings than in non-AMF seedlings, which is consistent with the results of previous studies on Hyacinths orientalis (Xie and $\mathrm{Wu}, 2018$ ), Phaseolus radiatus (Bhattacharjee and Sharma, 2012), and onions (Perner et al., 2008), indicating that AMF infection is conducive to the accumulation of pigment and the enhancement of photosynthesis.

The ratio of chlorophyll to flavonoids is the nitrogen balance index, which is an important indicator of the nitrogen status in plants and reflects the growth of plants and the degree of absorption and utilization of proteins by the organism (Dong et al., 2020). DS can inhibit the absorption of nitrogen by plants, resulting in insufficient nutrient supply in plants (Sarhadi et al., 2020), while AMF 


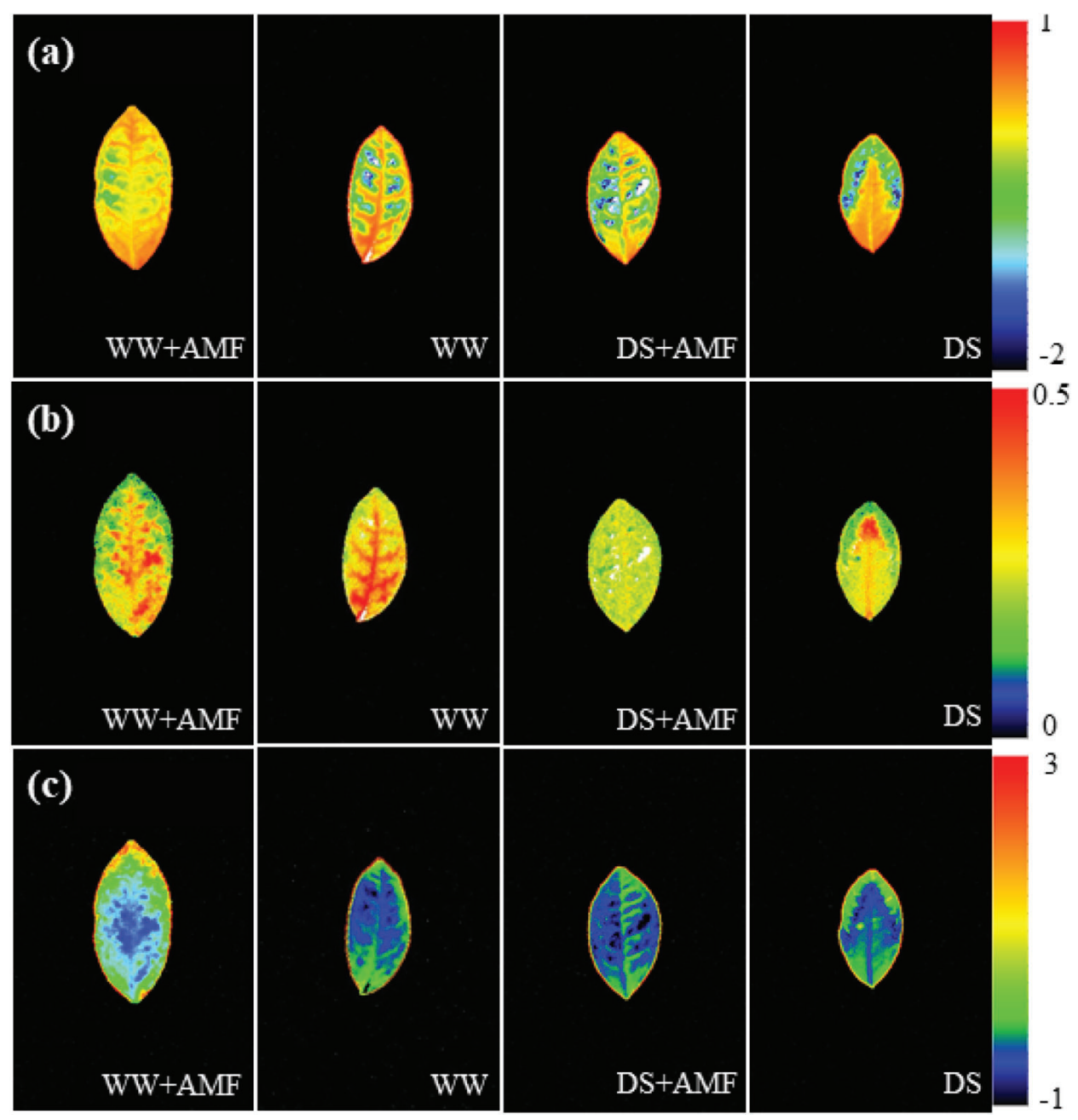

FIGURE 5. Effect of AMF on chlorophyll fluorescence parameters of tea seedlings (Camellia sinensis 'Fuding Dabaicha') under drought stress. (a) QY_max (b) QY_lss (c) NPQ_lss. can improve the absorption of nitrogen and maintain normal nutrient supply (He et al., 2018). This study demonstrated that DS reduced the nitrogen absorption of tea seedlings, whereas AMF inoculation significantly improved the plant nitrogen balance index, with the positive effect being more significant under WW conditions. This is mainly attributed to the enhancement of nutrient absorption by roots and the improvement of plant nutritional status by AMF extraradical hyphae (He et al., 2018).

Water is essential for photosynthesis, and water deficiency significantly decreases leaf $P n, C i, G s$, and $T r$, severely affecting the ability of a plant to photosynthesize (Chandrasekaran et al., 2019). Mycorrhizal colonization is often associated with changes in gas exchange in the host plant (Zhu et al., 2011). In the present study, C. etunicatum inoculation significantly increased photosynthetic parameters under both WW and DS conditions. These results are in accordance with the findings of Wang et al. (2017) on trifoliate orange colonized by Funneliformis mosseae and Zhu et al. (2011) in hightemperature-stressed maize (Zea mays) plants colonized by G. etunicatum. These results further implied that AMF symbiosis enhances gas exchange capacity by reducing stomatal resistance and increasing transpiration flux (Zhu et al., 2011). Moreover, AMF can improve the photosynthetic capacity of host plants, which may be related to enhanced nutrient absorption, as previous studies have reported that an increase in $\mathrm{N}$ and $\mathrm{P}$ content can improve the stomatal conductance, leaf water potential, chlorophyll content, and photosynthetic rate, thereby improving the drought resistance of host plants (Ruiz-Sánchez et al., 2010). Furthermore, higher $\mathrm{Ci}$ concentrations appeared in AMF seedlings, irrespective of the soil water status, which may be because AMF reduce the damage to the photosynthetic apparatus (Mathur et al., 2019; Wang et al., 2017).

The light absorbed by plants is divided into three parts, of which chlorophyll fluorescence is emitted in the form of fluorescence, which reflects the photosynthetic capacity and resistance of plants in adverse environments (Papageorgiou and Govindjee, 2004). Plant chlorophyll fluorescence parameters reflect the chemical properties of plant photosynthetic reactor reaction centers, including QY-max, QY-Lss, and NQP_Lss, among which the most common parameter is the QY-max (Papageorgiou and Govindjee, 2004). Generally, DS can reduce the maximum photochemical efficiency, actual photochemical quantum yield, and photochemical quenching coefficient of leaves, and gradually increase with an increase in drought degree (Chen et al., 2016), while AMF inoculation can improve the chlorophyll fluorescence parameters (Jiang et al., 2019). This study suggests that DS markedly decreased the QY-max and QY-Lss regardless of AMF inoculated or not, which is consistent with the results of Ahmed et al. (2002) on mungbean subjected to water stress, further demonstrated that DS could inhibit the photorespiration of tea plants and reduce the activity of the photosynthetic apparatus (Miyashita et al., 2005). 
Nevertheless, AMF-colonized seedlings showed higher QY-max and QY-Lss and lower NQP_Lss than non-AMF-colonized seedlings, which is consistent with the results of Ren et al. (2019) on Sesbania rostrata. QY_max and QY_Lss are sensitive indices of photosynthetic performance. QY_Lss releases excessive excitation energy in the form of heat energy; therefore, the results of this study indicate that AMF inoculation can not only significantly improve the efficiency of light energy conversion in plant leaves and the electron transfer activity of the reaction center but could also significantly reduce the loss of light energy in the form of heat, so as to ensure the maximum accumulation of photosynthetic products in plants.

In short, AMF tea seedlings had better growth performance under DS conditions, which associated with the improved leaf moisture status by mycorrhizal widespread hyphae. Colonization by mycorrhizal fungi also induces greater photosynthesis, which is possibly involved in pigment accumulation, gas exchange and chlorophyll fluorescence amelioration.

Acknowledgement: The authors would like to extend their sincere appreciation to the Researchers Supporting Project Number (RSP-2021/134), King Saud University, Riyadh, Saudi Arabia.

Availability of Data and Materials: The data that support the findings of this study are available with the first authors and will be made available upon reasonable request.

Author Contribution: FJD, ZYR, QSW and CYL conceived and designed the study. FJD and ZYR performed the experiments and interpreted the data. CYL wrote the manuscript, and SRL, QSW and EFAA provided critical revision of the manuscript for important intellectual content. All authors commented on the results and the manuscript.

Funding Statement: This work was supported by the Open Fund of State Key Laboratory of Tea Plant Biology and Utilization (SKLTOF20200122).

Conflicts of Interest: The authors declare that they have no conflicts of interest to report regarding the present study.

\section{References}

Ahmed S, Nawata E, Hosokawa M, Domae Y, Sakuratani T (2002). Alterations in photosynthesis and some antioxidant enzymatic activities of mungbean subjected to waterlogging. Plant Science 163: 117-123.

Bethlenfalvay GJ, Ames RN (1987). Comparison of two methods for quantifying extraradical mycelium of vesicular-arbuscular mycorrhizal fungi. Soil Science Society of America Journal 51: 834-837.

Bhattacharjee S, Sharma GD (2012). Effect of dual inoculation of arbuscular mycorrhiza and rhizobium on the chlorophyll, nitrogen and phosphorus contents of pigeon pea (Cajanus cajan L.). Scientific Research 2: 25945.

Cao JL, Shao YD, Zou YN, Wu QS, Yang TY et al. (2021). Inoculation with Clariodeoglomus etunicatum improves leaf food quality of tea exposed to $\mathrm{P}$ stress. Notulae Botanicae Horti Agrobotanici Cluj-Napoca 49: 12166.

Chandrasekaran M, Chanratana M, Kim K, Seshadri S, Sa T (2019). Impact of arbuscular mycorrhizal fungi on photosynthesis, water status, and gas exchange of plants under salt stress-A meta-analysis. Frontiers in Plant Science 10: 457.

Chen BW, Qin ZH, Zhang Y, Xiao YF, Zhang XN et al. (2019). Dynamic changes of physiological activities and endogenous hormones in Melaleuca alternifolia under drought stress. Shandong Agricultural Sciences 51: 55-59 (in Chinese with English abstract).

Chen Y, Liu L, Guo Q, Zhu Z, Zhang L (2016). Effects of different water management options and fertilizer supply on photosynthesis, fluorescence parameters and water use efficiency of Prunella vulgaris seedlings. Biological Research 49: e66259. DOI 10.1186/s40659-016-0069-4.

Cheng HQ, Giri B, Wu QS, Zou YN, Kuča K (2021). Arbuscular mycorrhizal fungi mitigate drought stress in citrus by modulating root microenvironment. Archives of Agronomy and Soil Science 57: 1-12. DOI 10.1080/03650340.2021.1878497.

Cheruiyot EK, Mumera LM, Ng'etich WK, Hassanali A, Wachira FN (2009). High fertilizer rates increase susceptibility of tea to water stress. Journal of Plant Nutrition 33: 115-129. DOI 10.1080/01904160903392659.

Dong R, Miao Y, Wang X, Chen Z, Yuan F et al. (2020). Estimating plant nitrogen concentration of maize using a leaf fluorescence sensor across growth stages. Remote Sensing 12: 1139. DOI 10.3390/rs12071139.

Gupta S, Bharalee R, Bhorali P, Das SK, Bhagawati P et al. (2013). Molecular analysis of drought tolerance in tea by cDNAAFLP based transcript profiling. Molecular Biotechnology 53: 237-248. DOI 10.1007/s12033-012-9517-8.

He JD, Dong T, Wu HH, Zou YN, Wu QS et al. (2019). Mycorrhizas induce diverse responses of root TIP aquaporin gene expression to drought stress in trifoliate orange. Scientia Horticulturae 243: 64-69. DOI 10.1016/j.scienta.2018.08.010.

He X, Chen Y, Liu S, Gunina A, Wang X et al. (2018). Cooperation of earthworm and arbuscular mycorrhizae enhanced plant $\mathrm{N}$ uptake by balancing absorption and supply of ammonia. Soil Biology and Biochemistry 116: 351-359. DOI 10.1016/j. soilbio.2017.10.038.

Lin S, Lin J, Liu Q, Ai Y, Ke Y et al. (2014). Time-course of photosynthesis and non-structural carbon compounds in the leaves of tea plants (Camellia sinensis L.) in response to deficit irrigation. Agricultural Water Management 144: 98106. DOI 10.1016/j.agwat.2014.06.005.

Liu CY, Wang YJ, Wu QS, Yang TY, Kuča K (2020). Arbuscular mycorrhizal fungi improve the antioxidant capacity of tea (Camellia sinensis) seedlings under drought stress. Notulae Botanicae Horti Agrobotanici Cluj-Napoca 48: 1993-2005. DOI $10.15835 /$ nbha48412066.

Jiang Y, Liu XS, Li J, Yang JS, Jiang Y et al. (2019). Effects of arbuscular mycorrhizal fungi on the photosynthetic characteristics and fluorescence parameters of Tectona grandis seedlings under drought stress. Journal of Forest and Environment 39: 608615 (in Chinese with English abstract).

Mathur S, Tomar RS, Jajoo A (2019). Arbuscular Mycorrhizal fungi (AMF) protects photosynthetic apparatus of wheat under drought stress. Photosynthesis Research 139: 227-238. DOI 10.1007/s11120-018-0538-4.

Meng LL, He JD, Zou YN, Wu QS, Kuča K (2020). Mycorrhizareleased glomalin-related soil protein fractions contribute to soil total nitrogen in trifoliate orange. Plant, Soil and Environment 66: 183-189. DOI 10.17221/100/2020-PSE.

Miyashita K, Tanakamaru S, Maitani T, Kimura K (2005). Recovery responses of photosynthesis, transpiration, and stomatal 
conductance in kidney bean following drought stress. Environmental and Experimental Botany 53: 205-214. DOI 10.1016/j.envexpbot.2004.03.015.

Papageorgiou GC, Govindjee R (2004). Chlorophyll A fluorescence: A Signature of Photosynthesis. Dordrecht: Springer.

Perner H, Rohn S, Driemel G, Batt N, Schwarz D et al. (2008). Effect of nitrogen species supply and mycorrhizal colonization on organosulfur and phenolic compounds in onions. Journal of Agricultural and Food Chemistry 56: 3538-3545. DOI 10.1021/jf073337u.

Phillips JM, Hayman D (1970). Improved procedures for clearing roots and staining parasitic and vesicular-arbuscular mycorrhizal fungi for rapid assessment of infection. Transactions of the British Mycological Society 55: 158-161.

Qian W, Hu J, Zhang X, Zhao L, Wang Y et al. (2018). Response of tea plants to drought stress. Stress physiology of tea in the face of climate change. Singapore: Springer.

Quiroga G, Erice G, Aroca R, Zamarreño ÁM, García-Mina JM et al. (2020). Radial water transport in arbuscular mycorrhizal maize plants under drought stress conditions is affected by indole-acetic acid (IAA) application. Journal of Plant Physiology 246: 153115.

Ren CG, Kong CC, Wang SX, Xie ZH (2019). Enhanced phytoremediation of uranium-contaminated soils by arbuscular mycorrhiza and rhizobium. Chemosphere 217: 773-779.

Ruiz-Sánchez M, Aroca R, Muñoz Y, Polón R, Ruiz-Lozano JM (2010). The arbuscular mycorrhizal symbiosis enhances the photosynthetic efficiency and the antioxidative response of rice plants subjected to drought stress. Journal of Plant Physiology 167: 862-869.

Saboor A, Ali MA, Hussain S, El Enshasy HA, Hussain S et al. (2021). Zinc nutrition and arbuscular mycorrhizal symbiosis effects on maize (Zea mays L.) growth and productivity. Saudi Journal of Biological Sciences in press 28: 6339-6351.

Sarhadi H, Daneshian J, Valadabadi SA, Sharafabad HH, Afsharmanesh G (2020). The effect of drought stress and nitrogen fertilization on the active ingredient, chlorophyll $\mathrm{A}$ and B, and chlorophyll index of henna. Nexo Revista Científica 33: 694-706. DOI 10.5377/nexo.v33i02.10802.

Shao YD, Hu XC, Wu QS, Yang TY, Srivastava A et al. (2021). Mycorrhizas promote $\mathrm{P}$ acquisition of tea plants through changes in root morphology and $\mathrm{P}$ transporter gene expression. South African Journal of Botany 137: 455-462. DOI 10.1016/j.sajb.2020.11.028.

Shao YD, Zhang DJ, Hu XC, Wu QS, Jiang CJ et al. (2019). Arbuscular mycorrhiza improves leaf food quality of tea plants. Notulae Botanicae Horti Agrobotanici Cluj-Napoca 47: 608-614. DOI 10.15835/nbha47311434.
Shao YD, Zhang DJ, Hu XC, Wu QS, Jiang CJ et al. (2018). Mycorrhiza-induced changes in root growth and nutrient absorption of tea plants. Plant, Soil and Environment 64: 283-289. DOI 10.17221/126/2018-PSE.

Tohge T, De Souza LP, Fernie AR (2017). Current understanding of the pathways of flavonoid biosynthesis in model and crop plants. Journal of Experimental Botany 68: 4013-4028. DOI 10.1093/jxb/erx177.

Tyagi J, Varma A, Pudake RN (2017). Evaluation of comparative effects of arbuscular mycorrhiza (Rhizophagus intraradices) and endophyte (Piriformospora indica) association with finger millet (Eleusine coracana) under drought stress. European Journal of Soil Biology 81: 1-10.

Upadhyaya H, Panda SK (2013). Abiotic stress responses in tea [Camellia sinensis L (O) Kuntze]: An overview. Reviews in Agricultural Science 1: 1-10.

Wang MH, Ding D, Zhang CY, Gao XZ (2020). Effects of drought stress on growth and chlorophyll fluorescence characteristics of tea seedlings. Journal of Tea Science 40: 478-491 (in Chinese with English abstract).

Wang WX, Zhang F, Chen ZL, Liu J, Guo C et al. (2017). Responses of phytohormones and gas exchange to mycorrhizal colonization in trifoliate orange subjected to drought stress. Archives of Agronomy and Soil Science 63: 14-23.

Wu QS, Liu CY, Zhang DJ, Zou YN, He XH et al. (2016). Mycorrhiza alters the profile of root hairs in trifoliate orange. Mycorrhiza 26: 237-247.

Wu QS, Shao YD, Gao XB, Xia TJ, Kuča K (2019). Characterization of AMF-diversity of endosphere versus rhizosphere of tea (Camellia sinensis) crops. Indian Journal of Agricultural Sciences 89: 348-352.

Xie MM, Wu QS (2018). Arbuscular mycorrhizal fungi regulate flowering of Hyacinths orientalis L. Anna marie. Emirates Journal of Food and Agriculture 30: 144-149.

Xie Y, Yang L, He Z (2019). Effects of AMF infection on photosynthetic characteristics of tomato under salt stress. IOP Conference Series: Earth and Environmental Science, Vol. 295, No. 2, pp. 012077. IOP Publishing.

Zhang F, Zou YN, Wu QS (2018). Quantitative estimation of water uptake by mycorrhizal extraradical hyphae in citrus under drought stress. Scientia Horticulturae 229: 132-136.

Zhu XC, Song FB, Liu SQ, Liu TD (2011). Effects of arbuscular mycorrhizal fungus on photosynthesis and water status of maize under high temperature stress. Plant and Soil 346: 189-199.

Zou YN, Zhang F, Srivastava AK, Wu QS, Kuča K (2021). Arbuscular mycorrhizal fungi regulate polyamine homeostasis in roots of trifoliate orange for improved adaptation to soil moisture deficit stress. Frontiers in Plant Science 11: 2046. 